\title{
Polymorphism in Giemsa Bandlng Patterns in Allium sativum
}

\author{
Satyesh Chandra Roy \\ Chromosome Research Centre, Department of Botany, University of Calcutta, \\ 35, Ballygunge Circular Road, Calcutta-700019, India
}

Received June 19, 1976

The structural heterozygosity of the chromosome complement has been noted in different populations of Allium sativum by different authors (Battaglia 1963, Sen 1973).

The chromosome complement of $A$. sativum is $2 n=16$. It consists of six pairs of metacentric chromosomes and two pairs with secondary constrictions. The different populations of this species are claimed to have a distinct structural heterozygosity in relation to chromosomes with four secondary constrictions. The other chromosomes too, cannot be strictly paired (Sen 1973). The four chromosomes with secondary constriction can also be easily identified because all the other chromosomes are metacentric. On the basis of this data, the present work was undertaken to utilize Giemsa banding technique, as a tool to investigate the structural heterozygosity in this species.

\section{Material and method}

The different commercial cultivars of garlic, A. sativum were used. Healthy growing root tips were pretreated in 0.05 percent colchicine at room temperature for 4 hours and then fixed for overnight in a mixture of acetic-alcohol (1:3). The fixed roots were hydrolysed in $(\mathrm{N}) \mathrm{HCl}$ for 30 seconds to one minute and squashed in $45 \%$ acetic acid on a clean slide. The cover glass was coated previously with a thin layer of glycerine albumen. The cover glasses were detached and air dried. They were then treated with saturated solution of barium hydroxide for 5 minutes at room temperature, were thoroughly washed in distilled water and then incubated in $2 \times \mathrm{SSC}$ at $60^{\circ} \mathrm{C}$ for 2 hours. The cover glasses were rinsed in distilled water, stained in 5\% Giemsa solution in phosphate buffer at pH 6.8 for 20-30 minutes, air dried and mounted in Euparal. The staining technique of Vosa and Marchi was followed with slight modifications (Vosa and Marchi 1972). Normal chromosome preparations were made with standard aceto-orcein staining technique (Fig. 1).

All the photographs were taken in Zeiss Ultraphol II microscope with an ORWO NP 22 films.

\section{Results}

Chromosome preparations from root tips of 30 bulbs studied showed almost 
identical banding pattern in each population. The four chromosomes with secondary constrictions showed distinct bands, except in one case where only two of these four chromosomes revealed bands. The metacentric chromosomes did not show

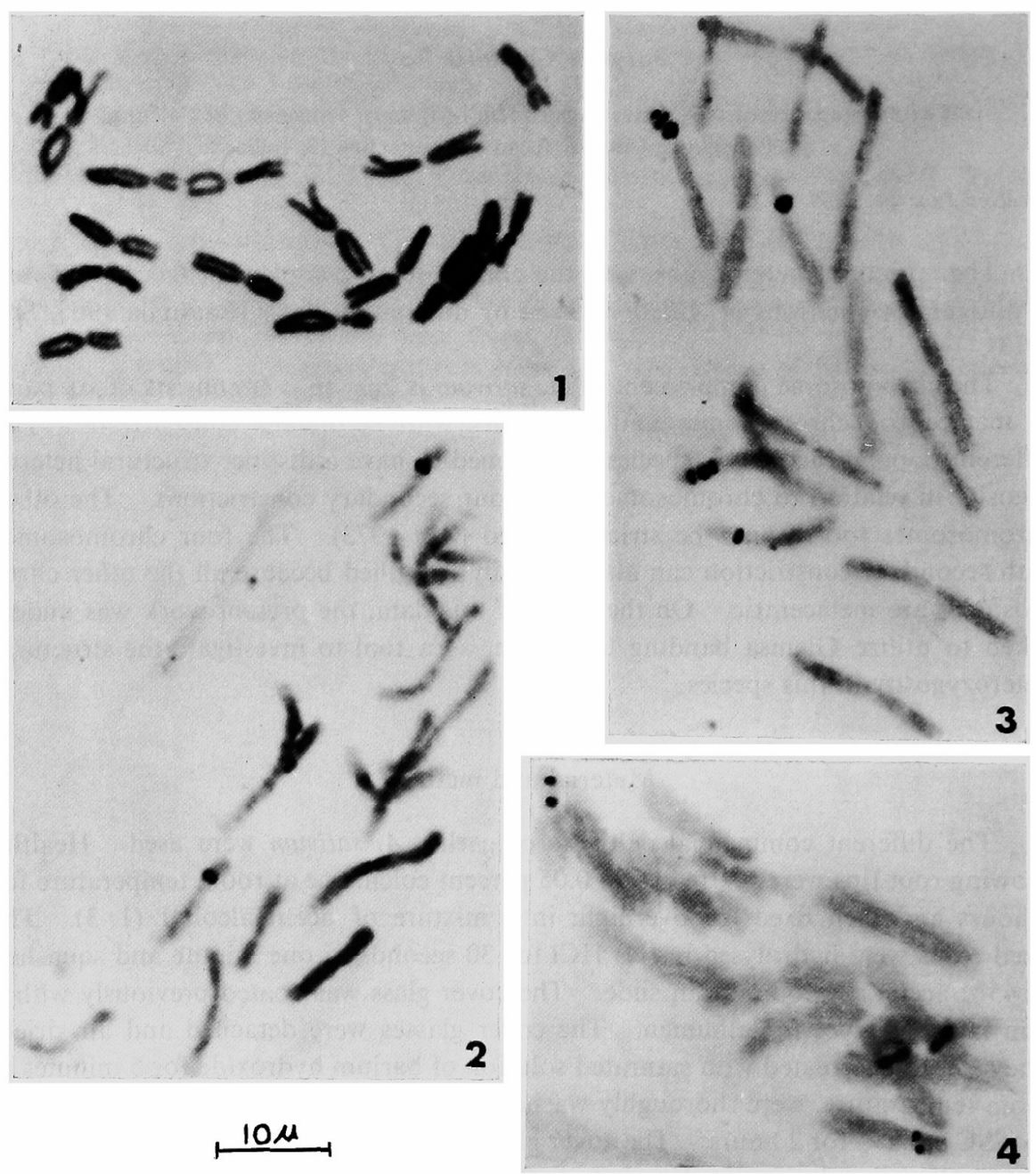

Figs. 1-4. 1, somatic plate showing $2 n=16$ chromosomes following orcein squash. 2, cell showing two distinct bands in two chromosomes after Giemsa staining. 3, showing bands in four chromosomes with heteromorphic nature in one pair only. 4, two chromosomes showing heteromorphicity in having two bands in one metacentric chromosome and one in a chromosome with secondary constriction.

any banding at all except in one case. In all the populations studied, band adjacent to the secondary constriction was found in all the chromosomes in one case and only in one pair in other cases. No bands at the centromeric region was found with the help of this technique in this material. Based on the intensity of the colour and the constancy of its occurrence, the banding pattern as could be seen in individuals can 
be described as follows:

I. One distinct band was found in each of the two chromosomes with secondary constrictions at identical location (Fig. 2).

II. Banding is found in four chromosomes with secondary constrictions. Of them, two chromosomes show distinct bands which indicate their heterozygosity. In the short arm of one of the chromosomes (Fig. 3), the two comparatively faint bands are located on both sides of the broad band whereas in the homologous one, the two faint bands are present one after another adjacent to the distinct broad band. The other two chromosomes have a single band in one arm, which are homomorphic in respect to the banding pattern.

III. It is similar to number one but shows heteromorphy in banding pattern having 2 bands in one metacentric chromosome and one band in one chromosome with secondary constriction (Fig. 4). These can be easily identified due to the fact that all the chromosomes excepting those with secondary constrictions are metacentric.

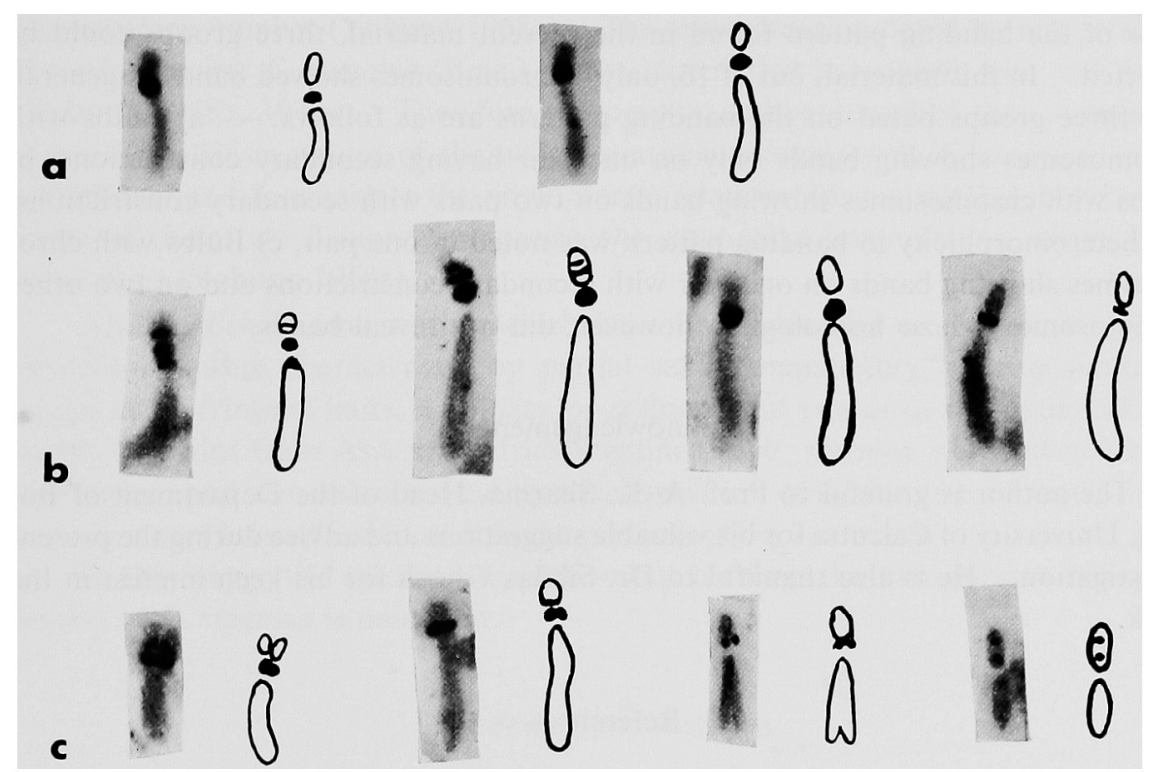

Fig. 5. a, two chromosomes with secondary constrictions showing banding pattern in the first population. b-c, showing heteromorphicity in banding pattern in the second population.

An analysis of the prophasic stages indicates that the number of bands found in metaphases is equal to that in interphase stages.

\section{Discussion}

Though the structural heterozygosity was noted by previous authors in almost all the chromosomes including those with secondary constriction the banding pattern reveals heterozygosity in all of the three populations studied with a somewhat different picture. No variation in morphological characters is reported with 
this structural heterozygosity. In the first type no heterozygosity is found. The second one shows heterozygosity in one pair only. The third type reveals heterozygosity in one metacentric chromosome and in one chromosome with secondary constriction. Very few attempt has been made to study this structural heterozygosity in chromosome complement by any special technique other than conventional orcein squashes. The structural heterozygosity in chromosomes of Leopoldia comosa of Liliaceae was revealed by Bentzer and Landstrom (1975) with the help of Giemsa staining. They found variation in banding pattern between homologous or analogous chromosomes within a species. The chemical nature of variation in banding pattern is yet to be established.

\section{Summary}

The structural variation in the chromosome complement of Allium sativum has been noted by previous workers. However, no attempt has been made to study the heterozygosity with the help of banding technique after Giemsa staining. On the basis of the banding pattern found in the present material, three groups could be detected. In this material, out of 16 , only 4 chromosomes showed bands in general. The three groups based on the banding patterns are as follows:- a) Bulbs with chromosomes showing bands only on one pair having secondary constrictions, b) Bulbs with chromosomes showing bands on two pairs with secondary constrictions, but heteromorphicity in banding pattern was noted in one pair, c) Bulbs with chromosomes showing bands on one pair with secondary constrictions and on two other chromosomes, whose homologues, however, did not reveal bands.

\section{Acknowledgements}

The author is grateful to Prof. A. K. Sharma, Head of the Department of Botany, University of Calcutta for his valuable suggestions and advice during the present investigation. He is also thankful to Dr. Sibdas Ghosh for his keen interest in the work.

\section{References}

Battaglia, E. 1963. Mutazione chromosomica e cariotipo fondamentale in Allium sativum. Caryologia (Firemze) 16: 1-46.

Bentzer, B, and Landstrom, T. 1975. Polymorphism in chromosomes of Leopoldia comosa (Liliaceae) revealed by Giemsa staining. Hereditas $80: 219-232$.

Sen, S. 1973. Structural hybridity intra and interspecific level in Liliales. Folia Biologica 21: $183-197$.

Vosa, C. G. and Marchi, P. 1972. Quinacrine fluorescence and Giemsa staining in plants. Nature New Biol. 237: 191-192. 